\title{
Correction to: Somatic symptom and related disorders in children and adolescents: evaluation of a naturalistic inpatient multidisciplinary treatment
}

Pola Heimann ${ }^{1}$, Beate Herpertz-Dahlmann ${ }^{1}$, Jonas Buning ${ }^{1}$, Norbert Wagner ${ }^{2}$, Claudia Stollbrink-Peschgens², Astrid Dempfle ${ }^{3}$ and Georg G. von Polier ${ }^{1 *}$

\section{Correction to:}

Child Adolesc Psychiatry Ment Health (2018) 12:34

https://doi.org/10.1186/s13034-018-0239-y

Following publication of the original article [1], the authors flagged an error in the "Authors' contributions" section of the article.

The (incorrect) text in this section says:

$\mathrm{BH}$ and JB conceptualised the study. JB supervised data collection, PH, AD and GGP analysed and interpreted the data and drafted the manuscript. BH, NW, and CS revised the manuscript critically. All authors read and approved the final manuscript.

As such, please be advised that the correct text ("correction in bold) is:

$\mathrm{BH}$ and JB conceptualised the study. JB supervised data collection, $\mathrm{PH}, \mathrm{AD}$ and GGP analysed and interpreted the data and $\mathbf{P H}$ drafted the manuscript. $\mathrm{BH}$, NW, and CS revised the manuscript critically. All authors read and approved the final manuscript.

\begin{abstract}
Author details
${ }^{1}$ Department of Child and Adolescent Psychiatry, Psychosomatics and Psychotherapy, RWTH Aachen University, Aachen, Germany. ${ }^{2}$ Department of Pediatrics, RWTH Aachen University, Aachen, Germany. ${ }^{3}$ Department of Medical Informatics and Statistic, University Schleswig-Holstein, Kiel, Germany.

The original article can be found online at https://doi.org/10.1186/s1303 4-018-0239-y.
\end{abstract}

\section{Publisher's Note}

Springer Nature remains neutral with regard to jurisdictional claims in published maps and institutional affiliations.

Published online: 01 November 2018

Reference

1. Heimann P, Herpertz-Dahlmann B, Buning J, Wagner N, StollbrinkPeschgens C, Dempfe A, von Polier GG. Somatic symptom and related disorders in children and adolescents: evaluation of a naturalistic inpatient multidisciplinary treatment. Child Adolesc Psychiatry Ment Health. 2018;12:34. https://doi.org/10.1186/s13034-018-0239-y.

\footnotetext{
*Correspondence: gvonpolier@ukaachen.de

${ }^{1}$ Department of Child and Adolescent Psychiatry, Psychosomatics

and Psychotherapy, RWTH Aachen University, Aachen, Germany

Full list of author information is available at the end of the article
} 